REVIEW

\title{
Congenital heart disease in pregnancy
}

\author{
C E G Head, S A Thorne
}

Postgrad Med J 2005;81:292-298. doi: 10.1136/pgmj.2004.026625

Congenital heart disease is now more prevalent than acquired in pregnancy in the developed world. In pregnancy the fall in systemic vascular resistance and increase in blood volume and cardiac output can cause functional deterioration in certain conditions. A minimally symptomatic woman with good ventricular function, normal oxygen saturation, and no left heart obstruction should tolerate pregnancy well. Women with pulmonary hypertension or dilated aortic root (pre-replacement) should be counselled against pregnancy and given appropriate contraceptive advice. The optimum management of a pregnant woman with a metallic prosthetic valve remains to be determined.

See end of article for authors' affiliations

Correspondence to:

Dr C Head, GUCH Office, 5 th Floor Jules Thorn Building, The Middlesex Hospital, Mortimer Street, London WIN 8AA, UK; cathy.head@uclh.nhs.uk

Submitted 9 August 2004 Accepted

6 September 2004
O $\mathrm{f}$ the 7 per 1000 live infants born with congenital heart disease, over $85 \%$ now survive into adult life. ${ }^{1}$ Continuing progress in medical and surgical management of paediatric congenital heart disease is likely to increase this proportion. The UK population of adults with congenital heart disease is increasing by 1600 cases with significant or complex disease per year. ${ }^{2}$ Many women in this group wish to have children, such that congenital heart disease is now more common than acquired in pregnancy in the developed world. ${ }^{3}$

In this article we review the haemodynamic changes of pregnancy and discuss pre-pregnancy risk stratification and subsequent management of the pregnant woman with congenital heart disease. Because in all but the lowest risk cases it is preferable to have a planned rather than accidental pregnancy, we also review current recommendations for contraception in this population.

\section{CARDIOVASCULAR CHANGES IN PREGNANCY}

Early in gestation the upregulation of nitric oxide synthesis by oestradiol causes arterial vasodilatation and a reduction in both systemic and pulmonary vascular resistance. Simultaneously the normal fall in heart rate at the end of the menstrual cycle fails to occur, and the heart rate increases by $10-20 \mathrm{bpm}$ for the duration of the pregnancy. The reduction in afterload and blood pressure stimulates an increase in plasma volume, and thus preload, by activation of the renin-angiotensin-aldosterone system. End diastolic volume, stroke volume, and contractility increase such that cardiac output reaches roughly $140 \%$ of prepregnancy level by 20 28 weeks gestation. Blood pressure (systolic and diastolic) gradually declines by about $10 \mathrm{~mm} \mathrm{Hg}$ to its nadir at about 20 weeks, before returning to prepregancy levels by term. See figure 1 and Nelson-Piercy, ${ }^{4}$ Hunter and Robson, ${ }^{5}$ Cutforth and MacDonald. ${ }^{6}$ It is important to note that although central venous pressure and pulmonary capillary wedge pressure remain unchanged, serum colloid osmotic pressure is reduced by the plasma expansion and the pregnant woman is therefore at increased risk of pulmonary oedema.

Labour, particularly the second stage, is associated with a further increase in cardiac output as pain increases heart rate via the sympathetic response and stroke volume is augmented by autotransfusion during contractions. In the absence of haemorrhage there is also significant autotransfusion after delivery. Thus the later stages of labour are a period of high risk for pulmonary oedema.

Structural changes to the heart and great vessels also occur. Echo diameters and orifice areas of all four valves increase, causing a higher incidence of valvular regurgitation. Changes in the extracellular matrix of the aortic media increase compliance but also, in combination with the increased cardiac output, the risk of dissection.

The examination of the normal cardiovascular system in pregnancy reflects these changes: $80 \%$ of healthy pregnant women have a third heart sound and $>90 \%$ a soft ejection systolic murmur at the left sternal edge.

\section{PRE-PREGNANCY ASSESSMENT AND RISK STRATIFICATION}

In all but the most straightforward cases a planned pregnancy is preferable to one unplanned. Before pregnancy a full clinical assessment should be made, including measurement of oxygen saturation, ECG, chest radiography, and echocardiogram. As pre-pregnancy functional capacity is an important predictor of a woman's ability to tolerate pregnancy, with those in NYHA classes I and II generally having a good outcome, treadmill exercise testing can be useful to define this. Invasive investigation may be indicated, for example for calculation of shunts or pulmonary vascular resistance. An estimate of maternal and fetal risk can then be given, together with recommendations for any medical, interventional, or surgical treatment before conception.

Abbreviations: $A S D$, atrial septal defect; $P F O$, patent foramen ovale; $\mathrm{AV}$, atrioventricular; CCTGA, congenitally corrected transposition of the great arteries 


\section{Box 1 Cardiovascular changes in pregnancy}

- Decreased systemic and pulmonary vascular resistance

- Increased heart rate

- Increased plasma volume therefore decreased packed cell volume/colloid osmotic pressure

- Increased end diastolic volume and cardiac output

- Third heart sound and ejection systolic murmur

\section{Maternal}

In parallel with the known lesion specific risks discussed below, a scoring system ${ }^{7}$ developed and validated from the follow up of 562 pregnant women with congenital or acquired cardiac disease receiving tertiary care predicts the risk of an adverse maternal event (pulmonary oedema, sustained arrhythmia, stroke, cardiac arrest, or cardiac death) by awarding one point for each of:

- Cyanosis (oxygen saturation $<90 \%$ ) or NYHA functional class $>$ II

- Left heart obstruction

- Systemic ventricular dysfunction $(\mathrm{EF}<40 \%)$

- Prior cardiac event (pulmonary oedema, arrhythmia, CVA/ TIA)

Adverse event rates are about $5 \%, 30 \%$, and $60 \%$ for 0,1 , and $>1$ points.

\section{Fetal}

In addition to the factors above, smoking, anticoagulation, and multiple pregnancy are adverse predictors of fetal and neonatal outcome, with the most common problems prematurity and low birth weight. Recurrence risk of any congenital heart disease (other than those with a known autosomal inheritance) is $3 \%-6 \%$ if the mother is affected, up to a 10 -fold increase over the general population. ${ }^{89}$ Families with multiple cases of congenital heart disease or lesions with specific inheritance such as Marfan syndrome should be offered referral to a clinical geneticist. Although a difficult issue to discuss, it is important that the prospective mother is fully aware of her expected lifespan and capacity.

\section{GENERAL PRINCIPLES \\ Antenatal care}

Women with a risk score of 0 and no additional lesion specific risk factors can generally be managed in their local hospital but in all other cases antenatal care should be shared with obstetric and anaesthetic colleagues experienced in high risk pregnancy, preferably in a combined clinic. Many cardiac drugs are relatively or absolutely contraindicated in pregnancy and treatment should be reviewed before conception. A detailed discussion of prescribing in pregnancy is beyond the scope of this article but useful information is available in the British National Formulary and Prescribing in Pregnancy. ${ }^{10}$ In general warfarin should be changed to subcutaneous low molecular weight heparin (with anti-Xa level monitoring) for the duration of pregnancy, except in the case of metallic valves discussed below.

\section{Labour and delivery}

Delivery for these women should usually occur at the tertiary centre with a written management plan in place well in advance. Although the American Heart Association guidelines ${ }^{11}$ do not advise antibiotic prophylaxis in non-instrumented vaginal or caesarean section delivery, in practice most

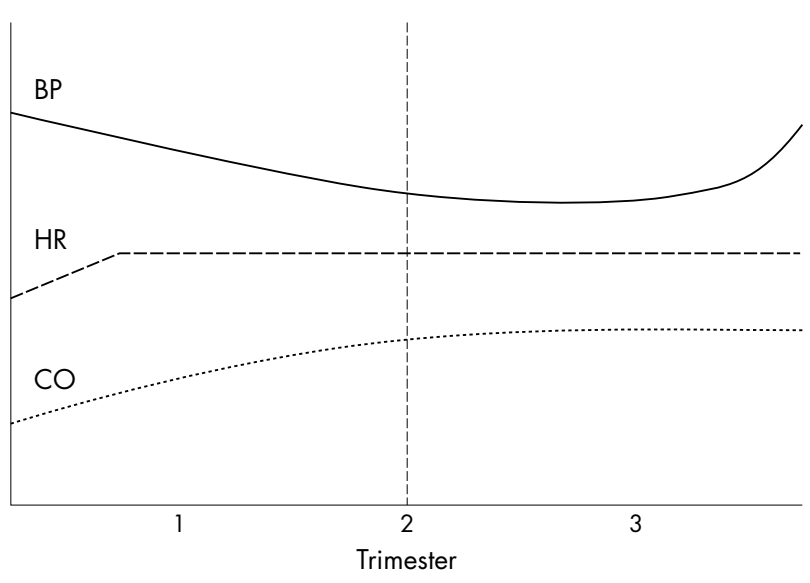

Figure 1 Representation of cardiovascular changes in pregnancy. HR, heart rate; $\mathrm{BP}$, blood pressure; $\mathrm{CO}$, cardiac output.

\section{Box 2 Predictors of poor pregnancy outcome}

- NYHA III or IV before pregnancy

- Saturation $<90 \%$ on air

- Left heart obstruction

- Previous cardiac event

- Systemic ventricular ejection fraction $<40 \%$

\section{Box 3 Cardiac indications for caesarean section}

- Aortopathy with root $>4 \mathrm{~cm}$

- Aortic dissection or aneurysm

- Warfarin treatment within two weeks

units give prophylaxis to all appropriate cases (that is, those who require it for dental treatment) at the onset of active labour to cover any unforeseen circumstances.

In the most cases vaginal delivery is recommended. Cardiac indications for caesarean section are Marfan syndrome with aortic root $>4 \mathrm{~cm}$, aortic dissection and warfarin treatment within the preceding two weeks-although the maternal INR may be normal the fetus clears warfarin more slowly and may still be at risk of cerebral haemorrhage. Low dose epidural anaesthesia does not cause excessive vasodilatation and with adequate volume expansion is the analgesia of choice. Invasive blood pressure monitoring is advisable in women with obstructive lesions (for example, aortic stenosis), in whom large fluid shifts may be poorly tolerated. Observation and monitoring in a high dependency unit may be required for up to a week post partum.

\section{SMALL LEFT TO RIGHT SHUNTS \\ Atrial septal defect}

In the presence of a normal pulmonary vascular resistance an unrepaired atrial septal defect (ASD) should be well tolerated. The pre-existing tendency to atrial arrhythmia may increase with the increase in cardiac output. The potential to shunt right to left in combination with the hypercoagulable state of pregnancy increases the risk of paradoxical embolism, especially with increases in 
intrathoracic pressure during labour. There should therefore be a low threshold for the use of compression stockings and prophylactic heparin in the presence of immobility or additional risk factors for venous thrombosis. This also applies to known patent foramen ovale (PFO). Surgical or device closure of the ASD removes this risk and if planned should therefore be carried out before pregnancy, although there is no evidence to support the same recommendation for PFO. There is no need for antibiotic prophylaxis in ASD other than for the first year after a device closure.

\section{Ventricular septal defect or patent ductus arteriosus}

A small defect with normal right sided pressures confers no added risk in pregnancy but antibiotic prophylaxis is required. Because of the large pressure gradient across the defect paradoxical embolism cannot occur. Large defects causing pulmonary vascular disease are discussed below under pulmonary hypertension.

\section{REGURGITANT VALVE LESIONS}

\section{Mitral valve prolapse and mitral regurgitation}

This is generally very well tolerated in pregnancy except if the mitral regurgitation is severe. Even in this case the offloading of the left ventricle caused by systemic vasodilatation is beneficial. Diuretics and vasodilators such as nitrates may be necessary in addition. Antibiotic prophylaxis is required.

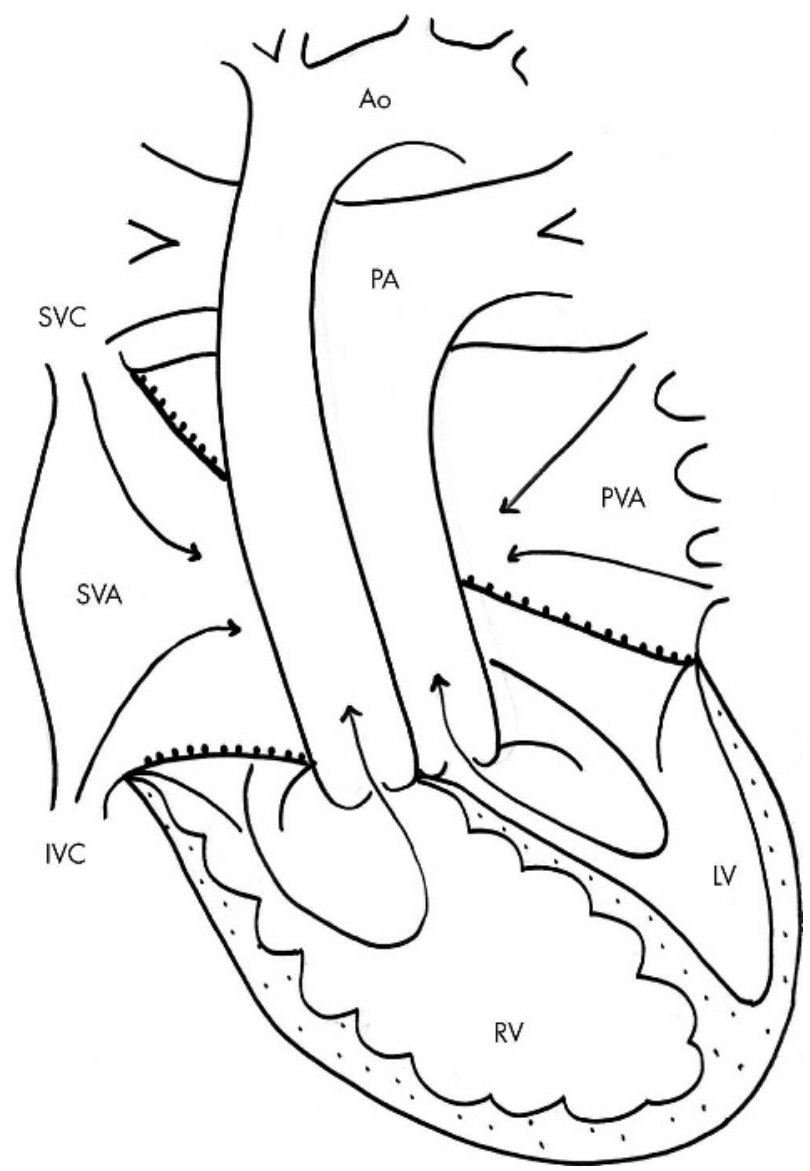

Figure 2 The Mustard operation for transposition of the great arteries IVC, inferior vena cava; SVC, superior vena cava; SVA, systemic venous atrium; PVA, pulmonary venous atrium; LV, left ventricle; RV, right ventricle; PA, pulmonary artery; Ao, aorta. The baffle re-routing the venous flow is marked as a suture line.

\section{Aortic regurgitation}

Similarly the physiological vasodilatation is beneficial but additional treatment may be necessary. ACE inhibitors are contraindicated in pregnancy. Antibiotic prophylaxis is required.

\section{OBSTRUCTIVE LESIONS}

Aortic stenosis and bicuspid aortic valve

Bicuspid aortic valve in the absence of any stenosis or root dilatation can be managed as a normal pregnancy with the addition of antibiotic prophylaxis. Aortic stenosis is well tolerated if the following conditions apply:

- Peak gradient $<80 \mathrm{~mm} \mathrm{Hg}$ and mean $<50 \mathrm{~mm} \mathrm{Hg}$ before pregnancy

- Normal left ventricular function

- Absence of symptoms

- Treadmill exercise test to target heart rate (220-age) with no ST change or arrhythmia and a normal haemodynamic response.

Otherwise aortic stenosis should be relieved before conception using balloon dilatation or a tissue valve if feasible, to avoid mechanical valve replacement. In a recent series of 49 pregnancies there was a $10 \%$ complication rate in women with peak gradient $>64 \mathrm{~mm} \mathrm{Hg}$ or valve area $<1 \mathrm{~cm}^{2}$ and no complications in those with less severe stenosis. ${ }^{12}$ The valve gradient will increase as pregnancy progresses and failure to do so is a warning sign of ventricular dysfunction. If a pregnant woman becomes or initially presents severely symptomatic there is benefit in bed rest and $\beta$ block but balloon valvuloplasty may need to be considered. Valve replacement during pregnancy carries a maternal mortality of $6 \%$ and fetal of $30 \% .{ }^{1}$

Delivery should be normal with antibiotic cover, avoiding vasodilatation and fluid shifts. It is unknown whether pregnancy accelerates the progression of congenital aortic stenosis.

\section{Coarctation of the aorta}

Pregnancy is low risk in repaired coarctation as long as there is no aneurysm at the site of repair. Magnetic resonance imaging or computed tomography should be performed before conception to ascertain this. The incidence of hypertension is higher than in the general pregnant population, particularly in those women with a residual gradient or unrepaired coarctation: $58 \%$ compared with $11 \%$ with gradient $<20 \mathrm{~mm} \mathrm{Hg}$ compared with 5\% general population. ${ }^{14}$ Whether or not there is a residual gradient blood pressure should be controlled to below 130/80 with $\beta$ blockers as the first line agent. In the presence of native coarctation, or re-coarctation, vaginal delivery with assisted second stage to avoid aortic wall stress is the method of choice. ${ }^{15}$ Caesarean section should be considered if there is an aneurysm. Mortality in a recent series (repaired and unrepaired) was $1 / 50$, the fatal aortic dissection occurring in a woman with Turner syndrome and a twin pregnancy who had undergone surgical repair in childhood. ${ }^{14}$ Angioplasty and stenting of coarctation during pregnancy and the puerperium is not recommended because of the increased predisposition to dissection during this period (see below). There are no series from which to estimate risk.

\section{Pulmonary stenosis}

This is generally well tolerated although in severe cases may precipitate right heart failure, tricuspid regurgitation, or atrial arrhythmia. Women with a pre-pregnancy peak to peak catheter gradient of $>50 \mathrm{~mm} \mathrm{Hg}$ or symptoms should be considered for balloon valvuloplasty or surgery pre-conception. 


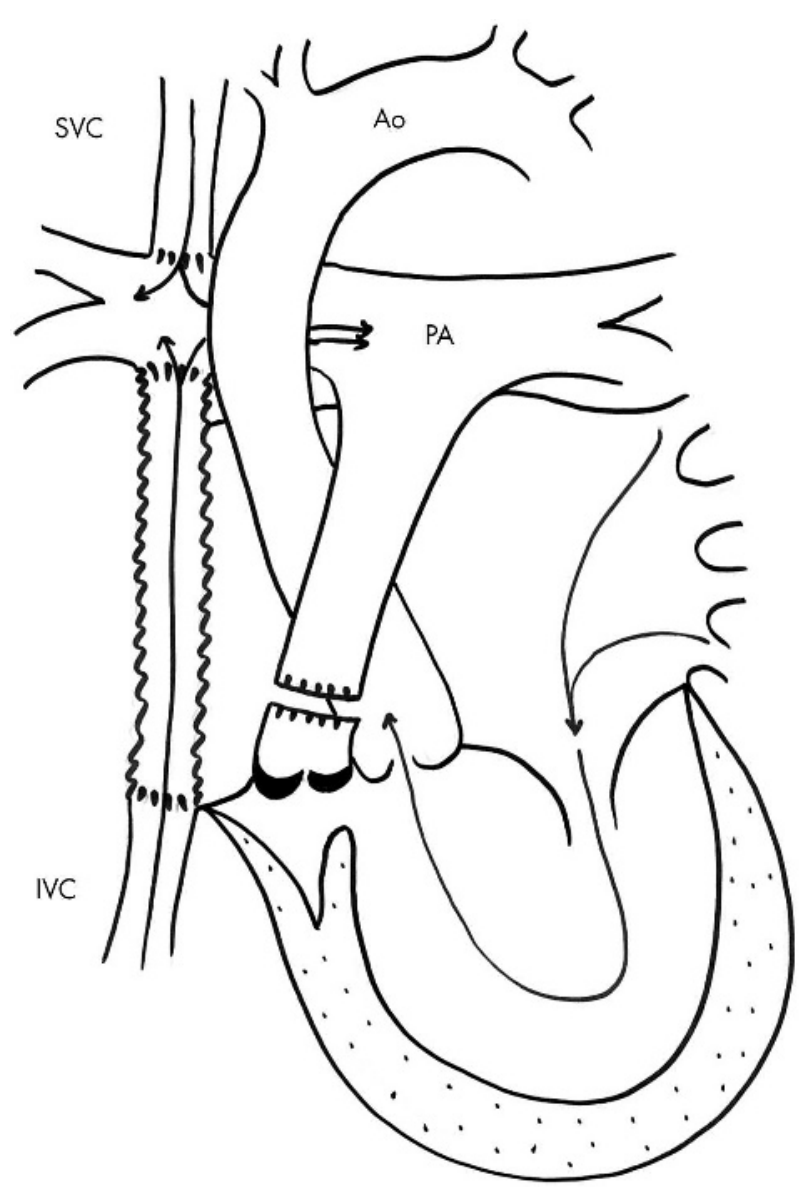

Figure 3 The total cavopulmonary connection (TCPC) variant of the Fontan operation for a single functional ventricle. Abbrieviations as figure 2. The SVC and IVC (via a conduit) are connected directly to the right pulmonary artery and the main pulmonary artery ligated. The ventricle supports the systemic circulation and there is no mixing. A rudimentary second ventricle can be seen.

Balloon valvuloplasty is also possible during pregnancy if symptoms develop. This should be carried out after the first trimester-the period of organogensis-with lead shielding of the abdomen and pelvis, transoesophgeal echo guidance, and low dose screening.

\section{DILATED AORTIC ROOT}

Aortic root dilatation secondary to cystic medial necrosis occurs in association with Marfan syndrome, familial thoracic aneurysm, bicuspid aortic valve, and repaired tetralogy of Fallot. It confers a risk of type A dissection, most commonly in the third trimester or immediately post partum - the time of greatest haemodynamic sheer stress to the aortic wall. Most of the literature concerns Marfan syndrome for which the overall mortality of pregnancy is $1 \%$, with the risk of aortic dissection increasing significantly if the aortic root diameter is greater than $4 \mathrm{~cm}$ or progressively dilating. ${ }^{16}$ These women should be advised to consider aortic root replacement before conception. This $4 \mathrm{~cm}$ cut off can be extrapolated to non-Marfan aortic root dilatation and echocardiograms should be performed before conception and every four to six weeks during pregnancy. ${ }^{17}$ It is probably sensible for all, and essential for the higher risk group, to be fully $\beta$ blocked throughout pregnancy. Low risk cases may have a normal delivery with an assisted second stage but most specialist units recommend caesarean section for higher risk women.
Box 4 High risk lesions, advise against

pregnancy

- Pulmonary hypertension

- Aortopathy with root $>4 \mathrm{~cm}$ or aneurysm, advise surgery first

- Severe aortic stenosis (peak gradient $>80 \mathrm{~mm} \mathrm{Hg}$ or symptoms), advise surgery first

- Systemic ventricular dysfunction NYHA III or IV symptoms

\section{PROSTHETIC VALVES}

Bioprosthetic valves are not associated with increased risk if there are no residual haemodynamic lesions and do not degenerate more rapidly in pregnancy as previously feared. ${ }^{18}$ Warfarin crosses the placenta, therefore the management of a mechanical prosthesis in pregnancy is far less straightforward and represents a conflict of interest between the mother and fetus. The safest option for the mother, to avoid valve thrombosis, is to remain taking warfarin for the duration of the pregnancy, stopping only for elective caesarean delivery at 38 weeks with intravenous heparin perioperatively. If vaginal delivery is planned warfarin should be changed to heparin at 36 weeks so the fetus has time to clear the warfarin. This strategy is associated with a $4 \%$ rate of maternal thromboembolism, a $6 \%$ rate of fetal abnormality, and a $30 \%$ rate of fetal loss. ${ }^{19}$ If the dose of warfarin required to achieve target INR is $<6 \mathrm{mg}$ daily the fetal complication rate is significantly reduced. ${ }^{20}$

Heparin does not cross the placenta: a strategy of substituting heparin for warfarin during the period of organogenesis (6-12 weeks) abolishes the risk of warfarin embryopathy but doubles the maternal thromboembolism rate to $9 \%$. Heparin throughout pregnancy is associated with a risk of thrombosis of up to $25 \%$, representing a range from the lower risk modern bileaflet valve in the aortic postion to the highest risk older (Starr-Edwards, Bjork-Shiley) valves in the mitral position. ${ }^{19}$ Fetal loss rates are similar for all methods of effective anticoagulation; this is thought to reflect placental haemorrhage. Each woman should be counselled as to the risks of each regimen so that she can make her choice.

\section{COMPLEX CONGENITAL HEART DISEASE Transposition of the great arteries (TGA) - after Mustard or Senning atrial repair}

Until about 20 years ago TGA correction was by Mustard or Senning atrial re-routing as shown in figure 2. These patients have a morphologically right ventricle as the systemic ventricle, with a high failure rate in the third and fourth decade. The ability to tolerate pregnancy depends largely on

\section{Box 5 Pregnancy with a metallic valve}

- Plan pregnancy before valve replacement if possible

- Fetal loss 30\% however anticoagulated

- Warfarin embryopathy only significant risk if daily dose $>5 \mathrm{mg}$ week 6-12

- Warfarin thrombotic risk $4 \%$

- Heparin then warfarin thrombotic risk $9 \%$

- Heparin thrombotic risk up to $25 \%$ 
the function of this systemic ventricle and its atrioventricular $(\mathrm{AV})$ valve. In the presence of good functional capacity before pregnancy (NYHA I or II), unobstruced venous pathways and systemic ventricular EF $>40 \%$ pregnancy should be low risk. In two small series (total of 20 women) there was only one case of significant deterioration in ventricular function, occurring in a woman NYHA II-III before pregnancy. ${ }^{21}{ }^{22}$ Atrial arrhythmia, also a feature of atrial repair, may become more common.

More recently, repair has been by the arterial switch operation, leaving the patient with the appropriate morphological ventricles but possible branch pulmonary artery stenoses. Pregnancy in theory should be well tolerated but this remains to be seen.

\section{Congenitally corrected transposition of the great arteries (CCTGA)}

CCTGA describes the combination of atrioventricular and ventriculoarterial discordance-that is, correctly connected circulations with a morphologically right systemic ventricle and left subpulmonary ventricle. Outcome depends on systemic ventricular function and the presence of associated lesions such as complete heart block, VSD, or pulmonary stenosis.

\section{Fontan operation for univentricular circulation}

The various forms of Fontan operation create two separate circulations in series in the presence of a functionally univentricular heart, see figure 3. These patients are therefore not cyanosed, but experience a long term low output state and are at risk of ventricular failure and atrial arrhythmia. They are generally anticoagulated with warfarin, which should be converted to full dose low molecular weight heparin for the duration of pregnancy. Maternal outcome again depends on functional capacity and ventricular function, which is more likely to be adequate if the single ventricle is morphologically left. If these are satisfactory and the woman accepts the $30 \%$ rate of first trimester fetal loss, ${ }^{23}$ twice that of the general population, then there is no reason to advise against pregnancy as has occurred in the past.

\section{Surgically corrected tetralogy of Fallot}

If these patients have good functional capacity pregnancy is not associated with any increased risk, even in the presence of significant pulmonary regurgitation.

\section{CYANOTIC HEART DISEASE WITHOUT PULMONARY HYPERTENSION}

Persisting cyanosis (unrepaired or palliated) is associated with a poorer outcome for both mother and fetus (see risk stratification above). The risk of paradoxical embolism should be reduced by appropriate hydration, mobilisation, and use of compression stockings. Because cyanosis also confers an increased bleeding tendency anticoagulants are not used routinely, but only if there is an additional indication. Increased right-left shunting can occur with the systemic vasodilatation of pregnancy, causing worsening cyanosis. Fetal outcome is dependent on maternal saturation-the chance of a live birth decreases from $92 \%$ with prepregnancy maternal saturation $>90 \%$ to $12 \%$ if maternal saturation is $<85 \%$. A significant proportion of these infants are premature or of low birth weight. ${ }^{24}$ Oxygen therapy may slightly improve maternal saturation by pulmonary vasodilatation and increased blood flow, but there is no evidence that this improves either maternal or fetal outcome.

\section{PULMONARY HYPERTENSION}

Pulmonary hypertension (PA systolic $>50 \mathrm{~mm} \mathrm{Hg}$ or $>2 / 3$ systemic) of any cause remains high risk for pregnancy. Patients with Eisenmenger syndrome have, by definition, suprasystemic PA pressures and have reversed their original left-right shunt. Maternal mortality remains at around $40 \%^{25}$ and avoidance of pregnancy or termination should be advised. Women who elect to continue should be admitted for bed rest from the third trimester and monitored for at least two weeks ante partum, as this is a period of continued risk of sudden death.

There are no controlled trials of the use of oxygen or anticoagulation but a Brazilian series of 13 pregnancies ${ }^{26}$ reported an improved maternal mortality (23\%) with a regimen of oxygen, heparin before delivery, and warfarin after 48 hours. Sixty per cent of infants were live born, most premature. Individual reports of the use of specific treatments to lower pulmonary artery pressure include intravenous prostacycline and inhaled nitric oxide but numbers are small. ${ }^{27}$ The existing right-left shunt is likely to increase with the fall in systemic vascular resistance and can be exacerbated further by hypovolaemia, so invasive blood pressure monitoring peripartum is required. There is no evidence to support the choice of either vaginal or caesarean delivery for cardiac reasons: vaginal delivery is associated with a lower average blood loss but also increased maternal effort. In practice early caesarean delivery is often required because of intrauterine growth retardation.

\section{CONTRACEPTION}

\section{Barrier methods}

These are safe for all cardiac patients and clearly have the added benefit of protection against sexually transmitted diseases. Reported failure rates are of the order of 3 per 100 woman years but with correct use are significantly lower than this.

\section{Hormonal methods}

The oestrogen component of the combined oral contraceptive (COCP) confers an increased risk of thrombosis. It is therefore contraindicated in women who already have a high thrombotic risk-that is, pulmonary hypertension, the Fontan circulation, or dilated cardiac chambers with the risk of atrial fibrillation-or in cyanosed patients in whom paradoxical embolism may occur. The standard progesterone-only "mini" pill is safe, but is less reliable than the COCP and is thus not the method of choice for women in whom avoidance of pregnancy is critical. Recommended progesterone-only preparations, more reliable as they act by suppression of ovulation, include the daily oral Cerazette (desogestrel), three monthly depot medroxyprogesterone, and the subcutaneous implant Implanon. Implanon is the method of choice for complex congenital heart disease as it is safe, highly effective and, in contrast with the IUCD, has no potential for significant complication associated with its insertion.

\section{Intrauterine devices}

These are not contraindicated but insertion of the device can be associated with bacteraemia and a vasovagal response. Antibiotic prophylaxis protects against endocarditis but a vasovagal can be life threatening in a haemodynamically unstable patient such as those with a Fontan circulation or Eisenmenger syndrome. Thus, if in doubt, the IUCD is best avoided.

\section{Sterilisation}

Sterilisation by tubal ligation may be appropriate for women in whom pregnancy would be high risk. The surgery itself however is not trivial, especially in women at risk of paradoxical embolism, as it includes a head down tilt and distension of the abdomen with carbon dioxide. It should therefore be carried out in a tertiary centre with consideration of cardiac anaesthesia. Sterilisation of the male partner is not 


\section{Box 6 Contraception}

- COCP contraindicated if tendency to thrombosis or cyanosed

- Cerazette and Implanon safe for all and highly effective

- IUCD needs antibiotic prophylaxis for insertion and beware vasovagal

generally advised if he has a much longer potential lifespan than his partner and may therefore wish to father children in a subsequent relationship.

\section{CONCLUSION}

Advances in paediatric cardiology and cardiac surgery are resulting in a growing population of young women with congenital heart disease well enough to contemplate pregnancy. Although certain conditions are still associated with a high maternal mortality, it is important to remember that inappropriate advice against pregnancy for a minor lesion can also be devastating to quality of life. The challenge to the cardiologist, obstetrician, and anaesthetist working as a team is to present the most realistic and comprehensible estimate of risk, both fetal and maternal, and then to achieve the best possible outcome of the patient's decision. Management by an experienced team in a designated specialist centre also facilitates the accumulation of an evidence base in a situation where individual conditions are rare.

\section{MULTIPLE CHOICE QUESTIONS (TRUE (T)/FALSE (F); ANSWERS AT END OF REFERENCES)}

1. The following are features of a normal pregnancy:

(A) Ejection systolic murmur

(B) Soft diastolic murmur

(C) Raised jugular venous pressure

(D) Hypertension

(E) Third heart sound

2. Maternal cyanosis is associated with an increased incidence of:

(A) Prematurity

(B) Fetal abnormality

(C) Fetal loss

(D) Peripartum haemorrhage

(E) CVA

3. The following are indications for elective caesarean section:

(A) Repaired coarctation of the aorta

(B) Metallic prosthetic valve on heparin

(C) Warfarin within two weeks

(D) Eisenmenger syndrome

(E) Dilated aortic root

4. Women with the following should be advised against pregnancy:

(A) Aortic stenosis peak gradient $50 \mathrm{~mm} \mathrm{Hg}$

(B) Marfan syndrome aortic root $5 \mathrm{~cm}$

(C) Eisenmenger syndrome

(D) Arterial switch operation

(E) Metallic aortic valve receiving warfarin $3 \mathrm{mg}$ daily
5. Indications for referral to a specialist centre for management of pregnancy include:

(A) Cyanosis

(B) Pulmonary stenosis gradient $30 \mathrm{~mm} \mathrm{Hg}$

(C) Previous TIA

(D) VSD with normal right sided pressures

(E) Mitral valve prolapse

6. The following conditions are matched with appropriate contraceptive choices:

(A) Eisenmenger syndrome and Implanon

(B) Severe LV dysfunction and sterilisation of the male partner

(C) Eisenmenger syndrome and the combined OCP

(D) Metallic valve and IUCD

(E) Metallic valve and tubal ligation

\section{Authors' affiliations}

C E G Head, S A Thorne, Department of Cardiology, University Hospital Birmingham, UK

Funding: none.

Competing interests: none.

\section{REFERENCES}

1 Perloff JK. Congenital heart disease in adults. A new cardiovascular subspecialty. Circulation 1991;84:1881-90.

2 Wren C, O'Sullivan JJ. Survival with congenital heart disease and need for follow up in adult life. Heart 2001;85:438-43.

3 Pitkin RM, Perloff JK, Koos BJ, et al. Pregnancy and congenital heart disease. Ann Intern Med 1990;1 12:445-54.

4 Nelson-Piercy C. Heart disease. In: Handbook of obstetric medicine. 2nd ed. London: Martin Dunitz, 2002:22-4.

5 Hunter S, Robson S. Adaptation of the cardiovascular system to pregnancy. In: Oakley C, ed. Heart disease in pregnancy. 1 st ed. London: BMJ Books, 1997:5-18

6 Cutforth R, MacDonald CB. Heart sounds and murmurs in pregnancy. Am Heart J 1966;71:741-7.

7 Siu SC, Sermer M, Colman JM, et al. Prospective multicenter study of pregnancy outcomes in women with heart disease. Circulation 2001;104:515-21.

8 Burn J, Brennan P, Little J, et al. Recurrence risks in offspring of adults with major heart defects: results from first cohort of British collaborative study. Lancet 1998;351:311-16.

9 Gill HK, Splitt M, Sharland GK, et al. Patterns of recurrence of congenital heart disease: an analysis of 6,640 consecutive pregnancies evaluated by detailed fetal echocardiography. J Am Coll Cardiol 2003;42:923-9.

10 Rubin P. Prescribing in pregnancy. 3rd ed. London: BMJ Books, 2000.

11 Dajani AS, Taubert KA, Wilson W, et al. Prevention of bacterial endocarditis. Recommendations by the American Heart Association. Circulation 1997;96:358-66.

12 Silversides CK, Colman JM, Sermer M, et al. Early and intermediate-term outcomes of pregnancy with congenital aortic stenosis. Am J Cardiol 2003;91:1386-9

13 Weiss BM, von Segesser LK, Alon E, et al. Outcome of cardiovascular surgery and pregnancy: a systematic review of the period 1984-1996. Am J Obstet Gynecol 1998; 179:1643-53.

14 Beauchesne LM, Connolly HM, Ammash NM, ef al. Coarctation of the aorta: outcome of pregnancy. J Am Coll Cardiol 2001;38:1728-33.

15 Yentis S, Gatzoulis MA, Steer P. Pregnancy and coarctation of the aorta. J R Soc Med 2003;96:471.

16 Lalchandani S, Wingfield M. Pregnancy in women with Marfan's syndrome. Eur J Obstet Gynecol Reprod Biol 2003;1 10:125-30.

17 Immer FF, Bansi AG, Immer-Bansi AS, et al. Aortic dissection in pregnancy: analysis of risk factors and outcome. Ann Thorac Surg 2003;76:309-14.

18 Avila WS, Rossi EG, Grinberg M, et al. Influence of pregnancy after bioprosthetic valve replacement in young women: a prospective five-year study. J Heart Valve Dis 2002;1 1:864-9.

19 Chan WS, Anand S, Ginsberg JS. Anticoagulation of pregnant women with mechanical heart valves: a systematic review of the literature. Arch Intern Med 2000;160:191-6.

20 Vitale N, De Feo M, De Santo LS, et al. Dose-dependent fetal complications of warfarin in pregnant women with mechanical heart valves. J Am Coll Cardiol 1999;33:1637-41.

21 Clarkson PM, Wilson NJ, Neutze JM, et al. Outcome of pregnancy after the Mustard operation for transposition of the great arteries with intact ventricular septum. J Am Coll Cardiol 1994;24:190-3.

22 Genoni M, Jenni R, Hoerstrup SP, et al. Pregnancy after atrial repair for transposition of the great arteries. Heart 1999;81:276-7. 
23 Canobbio MM, Mair DD, van der Velde $M$, et al. Pregnancy outcomes after the Fontan repair. J Am Coll Cardiol 1996;28:763-7.

24 Presbitero P, Somerville J, Stone S, et al. Pregnancy in cyanotic congenital heart disease. Outcome of mother and fetus. Circulation 1994:89:2673-6.

25 Yentis SM, Steer PJ, Plaat F. Eisenmenger's syndrome in pregnancy: maternal and fetal mortality in the 1990s. Br J Obstet Gynaecol 1998; 105:921-2.

26 Avila WS, Grinberg M, Snitcowsky R, et al. Maternal and fetal outcome in pregnant women with Eisenmenger's syndrome. Eur Heart $J$ $1995 ; 16: 460-4$.
27 Weiss BM, Hess OM. Pulmonary vascular disease and pregnancy: current controversies, management strategies, and perspectives. Eur Heart $J$ 2000;21:104-15

\section{ANSWERS}

1. (A) T, (B) F, (C) F, (D) F, (E), T; 2. (A) T, (B) F, (C) T, (D)

T, (E) T; 3. (A) F, (B) F, (C) T, (D) F, (E) T; 4. (A) F, (B) T, (C)

T, (D) F, (E) F; 5. (A) T, (B) F, (C) T, (D) F, (E) F. 6. (A) T,

(B) F, (C) F, (D) T, (E) T.

\section{WEB TRAWL}

The web trawl feature examines some of the web sites quoted in articles appearing in the journal. This month's column focuses on a single site for patients with multiple sclerosis.

- http://www.msdecisions.org.uk This site has been designed as a guide for multiple sclerosis patients who are being considered for treatment with a disease modifying drug (either beta interferon or glatiramer acetate). The site's aims, which are clearly stated on the home page, are to help patients decide whether to start on a course of treatment with one of these drugs, and, if they do go ahead, to help them decide which of the currently available drugs would be the best choice for them. A disclaimer reinforces that the site is not a substitute for medical advice, and that it is provided so that patients may be helped to make an informed choice regarding their future treatment; a decision that needs to be made in partnership with the team caring for them. From the home page, the user may access pages dealing with the disease itself, the treatments available, detailed information about disease modifying drugs, a decision making tool, and further reading. The pages are intended to be worked through in order ( 45 minutes is the minimum time recommended). The first section provides detailed background information on the different types of multiple sclerosis. It is clearly written and illustrated, with all medical terms explained. The next section outlines the treatment options available, and then continues with an in depth discussion of disease modifying drugs (DMDs), and the eligibility criteria for their use. Advice is also given for patients who are deemed ineligible for DMD treatment, so that they are able to consider other options. Following on from this, is a section detailing factors patients will need to consider if they start treatment with DMD, for example, will they be able to cope with injecting the drug themselves. Videos showing the injection process are provided, along with brief interviews with a number of patients who demonstrate different clinical scenarios. Next is the decision making tool, which consists of a number of highly subjective questions for the patient to answer on screen, at the end of which they are provided with a table showing the pros and cons of each treatment option. This table is very detailed, with colour coding to indicate the advantages and disadvantages of each DMD (and, indeed of having no treatment). This page could potentially be very confusing, but the user is able to hide some of the information, if there is an area they wish to concentrate on. The final section provides details of, and links to, other organisations that patients may find useful. The web site has been created with funding from the Department of Health, and was last updated on 31 January 2005, so the information provided is current. Patients with multiple sclerosis are likely to find it of great value in terms of the information provided, and indeed anyone who is not a neurologist and wishes a clear update on DMDs may also find the site of use. I would have no hesitation in recommending it.

Robyn Webber

Web Editor 